\title{
DECOMPOSIÇÃO E LIBERAÇÃO DE NUTRIENTES DO FOLHEDO DE ESPÉCIES FLORESTAIS NATIVAS EM PLANTIOS PUROS E MISTOS NO SUDESTE DA BAHIA ${ }^{(1)}$
}

\author{
A. C. GAMA-RODRIGUES ${ }^{(2)}$, N. F. BARROS ${ }^{(3)} \&$ M. L. SANTOS(4)
}

\begin{abstract}
RESUMO
As taxas de decomposição e os fluxos de nutrientes do folhedo de espécies florestais são distintos em plantios puros e mistos, porque são regulados não apenas pela qualidade do substrato, mas também pela qualidade do microambiente de acordo com o tipo de sistema de produção florestal. $O$ objetivo deste trabalho foi estimar as taxas de decomposição e a liberação de N e P do folhedo de espécies florestais em dois sistemas de plantio. Este trabalho foi desenvolvido em solos de tabuleiro do sudeste da Bahia, em plantios puros e mistos, com 22 anos de idade, de pau-roxo, Peltogyne angustiflora; putumuju, Centrol obi um robustum; arapati, Arapatiel la psi lophyl la; arapaçu, Scl erol obi um chrysophyllum; claraíba, Cordia trichotoma, e óleo-comumbá, Macrolobium latifolium. Também foram utilizadas uma floresta secundária, praticamente em estado clímax, e uma capoeira, de 40 anos de idade. A decomposição de folhedo, colocado em sacos de malha de $1 \mathrm{~mm}$, foi seguida durante um ano. 0 modelo que proporcionou melhor ajuste foi exponencial de $1^{\mathrm{a}}$ ordem. No plantio misto, as taxas de decomposição do folhedo do pau-roxo e óleo-comumbá foram significativamente superiores àquelas dessas espécies em seus plantios puros, ao contrário do observado para o arapati. No entanto, as taxas de decomposição do folhedo do putumuju, arapaçu e claraíba não foram alteradas significativamente. $O P$, e não o $N$, seria o nutriente mais limitante para a decomposição do folhedo dessas espécies. A liberação de $\mathbf{N}$ e $\mathbf{P}$ do folhedo de cada espécie variou de acordo com o microambiente. Dentre os ecossistemas heterogêneos, a taxa de decomposição do folhedo do plantio misto diferiu significativamente apenas da floresta natural. A maior liberação do $\mathbf{N}$ ocorreu no plantio misto; já para o $P$, a capoeira foi o ecossistema que apresentou significativamente a menor liberação. Conclui-se que o plantio mi sto proporciona
\end{abstract}

(1) Recebido para publicação em junho de 2001 e aprovado em agosto de 2003.

(2) Professor Associado do Laboratório de Solos, Centro de Ciências e Tecnologias Agropecuárias (CCTA), Universidade Estadual do Norte Fluminense - UE NF. CEP 28013-600 Campos dos Goytacazes (RJ ). Bolsista da FAPERJ . E-mail: tonygama@uenf.br

(3) Professor Titular do Departamento de Solos, Universidade Federal de Viçosa - UFV. CEP 36570-000 Viçosa (MG). Bolsista do CNPq. E-mail: nfbarros@ufv.br

(4) Doutorando no Laboratório de Solos, Centro de Ciências e Tecnologias Agropecuárias (CCTA), UENF. Bolsista FAPERJ . E-mail: luciomls@yahoo.com.br 


\begin{abstract}
maior capacidade em reciclar matéria orgânica e nutriente, o que indica serem os processos de decomposição e mineralização influenciados não apenas pela qualidade do substrato, mas também pela qualidade do microambiente. Assim, a extrapolação de resultados desses processos obtidos em cultivos monoespecíficos para sistemas florestais heterogêneos não seria apropriada.
\end{abstract}

Termos de indexação: floresta tropical, carbono, nitrogênio, fósforo, lignina.

\author{
SUMMARY: DECOMPOSITION AND NUTRIENT RELEASE FROM LEAF \\ LITTER IN PURE AND MIXED STANDS OF NATIVE TREE \\ SPECIES IN SOUTHEASTERN BAHIA STATE, BRAZIL
}

\begin{abstract}
Leaf litter decay rates and $\mathrm{N}$ and $\mathrm{P}$ fluxes of tree species are different in pure and mixed stands because the decomposition process is not only defined by the quality of the substrate, but of themicroenvi ronment (forest production systems) also. Theobjetive of this work was to estimate the decomposition rate and $\mathrm{N}$ and $\mathrm{P}$ release from leaf litter of native forest species planted under two different systems. Twenty two year-old mixed and pure stands of six hardwood species (Pel togyneangustiflora, Centrol obium robustum, Arapatiella psilophylla, Sclerolobium chrysophyllum, Cordia trichotoma, Macrolobium latifolium) native of the southeastern region of Bahia, Brazil, were evaluated. As reference for the analyzed characteristics, thestudy included a secondary forest near climax, and a 40- yearold naturally regenerated forest. Thedecompositi on of leaf litter in sacks of $1 \mathrm{~mm}$ mesh was observed during oneyear. Thesingleexponential model provided the best adjustment for all species in both planting systems (pure and mixed). Leaf litter decay rates of Peltogyne angustiflora and Macrolobium latifolium were significantly higher in the mixed than in the pure stand of the same species, unlike observed for Arapatiella psilophylla. The leaf litter decay rates of Centrolobium robustum, Sclerolobium chrysophyllum, and Cordia trichotoma were not altered significantly. Not N, but P limited the litter decomposition of thesespecies most. Therel ease of $\mathrm{N}$ and $\mathrm{P}$ from leaf litter of each species varied according to the mi croenvironment. Among the heterogeneous ecosystems, the leaf litter decay rate in mixed stands was theonly onethat differed si gnificantly from thenativeforest. Thehighest $\mathrm{N}$ releaseoccurred in themixed stand, whereas $\mathrm{P}$ rel easewas clearly lowest in theregenerated forest. I t was inferred that mi xed stands of forests species own a greater capability of recycling organic matter and nutrients. This indicates that the decay and mineralization processes arenot only influenced by thesubstratequality, but also thequality of themi croenvironment. I nformation about these processes obtained in pure stand systems should therefore not be extrapolated to heterogeneous forest systems.
\end{abstract}

Index terms: tropical forests, carbon, nitrogen, phosphorus, lignin.

\section{INTRODUÇÃO}

O processo de decomposição mantém a funcionalidade do ecossistema, possibilitando que parte do carbono incorporado na biomassa vegetal retorne à atmosfera como $\mathrm{CO}_{2}$ e outra parte, juntamente com os elementos minerais, seja incorporada ao solo (Olson, 1963; Odum, 1969). Esse processo é regulado pela interação de três grupos de variáveis: as condições físico-químicas do ambiente, as quais são control adas pel o clima e pelas características edáficas do sítio; a qualidade (orgânica enutricional) do substrato, que determina sua degradabilidade, e a natureza da comunidade decompositora (os macro e microrganismos) (Heal et al., 1997; Correia \& Andrade, 1999). A importância relativa de cada um desses três grupos de variáveis varia dentroe entresítios, resultando em um sistema hierárquico decontrole(Lavelleet al., 1993). Demodo geral, o clima controla o processo de decomposição em escala regional, enquanto a composição química domina o processo em escala local (Berg, 2000).

Assim, sob as mesmas condições edafoclimáticas, a taxa de decomposição do folhedo de diversas espécies florestais pode variar conforme a qualidade (teor de lignina, por exemplo) do substrato (Mellilo et al., 1982; Taylor et al., 1989). Dessa forma, o folhedo revelaria a capacidade diferenciada de espécies florestais em absorver ereciclar nutrientes, quando em sistemas puros. Entretanto, o uso dessas espécies em sistemas de plantio misto poderia resultar em rápida ciclagem de matéria orgânica e de nutrientes, mediante interações positivas dos fatores bióticos e abióticos que regulam o processo de decomposição do fol hedo de ecossistemas heterogêneos (Briones \& Ineson, 1996; GamaRodrigues, 1997; Gama-Rodrigues et al., 1999; Zimmer, 2002). Neste contexto, o plantio misto 
geraria um microambiente (interação de fatores físico-químicos e da biota decompositora) distinto daquele do plantio puro sob as mesmas condições edafoclimáticas do macroambiente.

A comparação da decomposição do fol hedo em povoamentos puros ou em misto e, ou, em floresta natural, permite avaliar possíveis alterações decorrentes detécnicas de manejo aplicadas einferir sobre a sustentabilidade das plantações. Em solos de baixa fertilidade, o acúmulo e a decomposição da serapilheira podem servir de indicadores de diferenças entre os dois sistemas, puro e misto, em especial no quetangeà disponi bilidade denutrientes para as plantas. Estudos desse tipo poderiam, ainda, fornecer subsídios para desenvol vimento de técnicas de manejo que sejam ecológica e economicamente viáveis.

A hipótese deste trabal ho considera que as taxas de decomposição e os fluxos de nutrientes dos folhedos das espécies florestais são distintos em plantios puros e mistos, porque são regulados não apenas pela qualidade do substrato, mas também pela qualidade do mi croambiente decorrente do tipo de sistema de produção florestal. Este trabal ho foi conduzido para testar esta hipótese.

\section{MATERIAL E MÉTODOS}

O trabalho foi realizado no arboreto da Estação E cológica do Pau-Brasil (ESPAB), Porto Seguro, Bahia ( $\left.16^{\circ} 23^{\prime} \mathrm{S} \mathrm{e} 39^{\circ} 11^{\prime} \mathrm{W}\right)$. A vegetação foi dassificada como "Floresta Ombrófila Densa das Terras Baixas"(Veloso et al., 1991). Estrutural efisionomicamente, essa vegetação muito seassemel ha à F loresta Amazônica (Mori \& Silva, 1980). O clima é classificado como Af (Vinha \& Lobão, 1989); durante o período experimental, a pluviosidade e a temperatura média mensal foram de $127 \mathrm{~mm}$ e de $24^{\circ} \mathrm{C}$, respectivamente (Quadro 1 ). O solo é um Argissolo Amarelo Tb álico, fortemente ácido e de fertilidadenatural muito baixa (Leão \& Melo, 1990).

Mediu-se a decomposição do folhedo de seis espécies nativas, plantadas em parcelas puras, sem adubação, no espaçamento de $2 \times 2 \mathrm{~m}$. As parcelas eram de $144 \mathrm{~m}^{2}$, totalizando 36 árvores por espécie. As espécies estudadas foram: pau-roxo, Peltogyne angustiflora Ducke; putumuju, Centrolobium robustum (Vell.) Mart.; arapati, Arapatiella psilophylla (Harms.) Cowan; arapaçu, Sclerol obium chrysophyllum Loep \& Endl; claraíba, Cordia trichotoma (Vell.) Arrab., e óleo-comumbá, Macrol obium Iatifol ium Vog.

A influência do microambiente (interação de fatores físi co-quími cos e da biota decompositora) na decomposição do fol hedo das espécies foi avaliada, colocando-seamostras deste material, coletadas nos plantios puros, sob as árvores do plantio misto, por um período de 360 dias. O plantio misto, além das espécies estudadas, era formado por outras 57 espécies nativas e exóticas, no mesmo espaçamento dos plantios puros, em parcela de $5.320 \mathrm{~m}^{2}$, totalizando 1.330 árvores plantadas aleatoriamentecom diferentes freqüências para cada espécie. A idade, em ambos os sistemas de plantio, era de 22 anos, na época das amostragens. A decomposição do fol hedo do plantio misto de uma Floresta Ombrófila Densa Secundária (com 93 espécies), praticamente em estado clímax, e de uma capoeira (com 37 espécies), deaproximadamente 40 anos de idade, foi determinada como referencial aos outros sistemas.

Uma porção do folhedo recém-caído, em novembro de 1994, foi col etada e col ocada em sacos de decomposição de $1 \mathrm{~mm}$ de malha (náilon), com dimensões de $20 \times 20 \mathrm{~cm}$ (Anderson \& Ingram, 1989). A massa seca referente ao tempo inicial foi obtida mediante um fator de correção após secagem de amostras em estufa a $75^{\circ} \mathrm{C}$ até peso constante. Para cada povoamento florestal, prepararam-se 20 sacos de decomposição, os quais for am col ocados sobre a serapilheira em cada situação. No período de 360 dias, foram coletados cinco sacos, a cada 90 dias, tendo sido as amostras limpas, para retirar o solo aderido, secas e pesadas.

Nas amostras, determinaram-se os teores de $P$ (colorimetricamente, pel o método do complexo fosfomolíbdico, reduzido com vitamina C, modificado por Braga \& Defelipo, 1974), após digestão nítricoperclórica, e de $\mathrm{N}$, pelo método Kjeldahl, descritos por Bataglia et al. (1983). O teor de C foi obtido por queima total de $1 \mathrm{~g}$ de material vegetal seco em estufa à $5{ }^{\circ} \mathrm{C}$, a $550{ }^{\circ} \mathrm{C}$, em mufla, considerando sua concentração como $500 \mathrm{~g} \mathrm{~kg}^{-1}$ da matéria orgânica

\section{Quadro 1. Preci pitação pluviométrica e temperatura mensal média do período de novembro de 1994 a novembro de 1995, na Estação E cológica do Pau-Brasil (BA)}

\begin{tabular}{lcc}
\hline \multicolumn{1}{c}{ Mês/ano } & Chuva & Temperatura \\
\hline & $\mathrm{mm}$ & ${ }^{\circ} \mathrm{C}$ \\
Novembro/94 & 202 & 23,3 \\
Dezembro/94 & 31 & 24,8 \\
J aneiro/95 & 52 & 25,4 \\
F evereiro/95 & 4 & 25,9 \\
Março/95 & 170 & 25,8 \\
Abril/95 & 266 & 24,7 \\
Maio/95 & 198 & 23,1 \\
J unho/95 & 136 & 21,4 \\
J ulho/95 & 242 & 21,2 \\
Agosto/95 & 70 & 20,9 \\
Setembro/95 & 65 & 21,5 \\
Outubro/95 & 100 & 23,6 \\
N ovembro/95 & 112 & 23,8 \\
\hline
\end{tabular}


livre de cinzas, conforme procedimento adotado por Schlesinger \& Hasey (1981), McClaugherty et al. (1985), Taylor et al. (1989) e van Wesemael (1993). $\mathrm{Na}$ determinação deligni na e de celul ose, empregouse o método da fibra em detergente ácido (FDA), de van Soest \& Wine (1968).

A matéria seca decomposta com o tempo foi calculada pela diferença entre o peso original ( $30 \mathrm{~g})$ e o peso determinado ao final de cada período de decomposição, obtendo-se, assim, o percentual depeso de material seco remanescente. Com esses dados estimaram-se as taxas da decomposição $(\mathrm{k})$ anual por diversos modelos de regressão comumente usados em estudos de decomposição (Wieder \& Lang, 1982). O model oque proporcionou melhor ajustepara todas as espécies em ambos os sistemas de plantio (puro e misto) foi a exponencial de $1^{\mathrm{a}}$ ordem, $\mathrm{Mt}=$ Mi e $\mathrm{e}^{-k T}$ (Olson, 1963); sendo Mt o percentual de peso de material seco remanescente após $\mathrm{t}$ anos e Mi $100 \%$ quando t é igual a zero.

Na comparação das taxas de decomposição das espécies entre os dois sistemas (puro e misto), utilizou-se o teste Student's t (Zar, 1996), análogo ao teste para comparação de médias entre duas populações. Este teste é baseado no erro-padrão da diferença entre os coeficientes de regressão (ß). Para comparar as taxas dentro dos sistemas, procedeuse primeiramente à análise de covariância (Zar, 1996) e, em seguida, caso necessário, aplicou-se o teste de Student's t.

Na análise de variância dos dados de percentual da quantidade de $\mathrm{N}$ ede $\mathrm{P}$ remanescentes no fol hedo, adotou-se o del ineamento inteiramentecasualizado, a exemplo do procedimento empregado por Wieder \& Lang (1982), considerando a ausência de ajustes significativos para um mesmo model o de regressão, para al gumas espécies, entre os sistemas de plantio.

\section{RESULTADOS E DISCUSSÃO}

A influência do microambiente sobre a perda de matéria seca do folhedo foi verificada para três das seis espécies florestais. No plantio misto, as taxas de decomposição do fol hedo do pau-roxo e do óleocomumbá foram significativamente superiores às do folhedo em seus plantios puros, ao contrário do observado para o arapati (Quadro 2). As taxas de decomposição do folhedo do putumuju, arapaçu e claraíba não foram alteradas significativamente. As espécies que mais distinguiram, ao longo do tempo, a influência dos dois sistemas de plantio (microambiente) foram oóleo-comumbá, cujo fol hedo apresentou aumento de $74,6 \%$ em sua taxa de decomposição no plantio misto, e o arapati, que, neste sistema de plantio, teveuma redução de 48,3 \% (Figura 1).

Em ambos os sistemas de plantio, houve diferenças significativas da taxa de decomposição entre as espécies florestais. As espécies que apresentaram a maior ea menor taxa de decomposição foram, respectivamente, a claraíba e o óleocomumbá, em plantios puros. No plantio misto, a claraíba também apresentou a maior taxa de decomposição, sendo a menor taxa observada para o arapati (Quadro 2).

As diferenças de taxas de decomposição do folhedo entre as espécies florestais em plantios puros expressariam o confundimento da interação microambiente-qualidade do substrato. Ou seja, cada espécie em plantio puro caracterizaria um tipo de microambiente produzindo folhedo de determinada qualidade. No entanto, no plantio misto, as diferenças de decomposição entre as espécies evidenciariam a importância da qualidade do substrato de cada espécie e, ou, do tipo e da

Quadro 2. Valores esti mados da taxa de decomposição (k) do fol hedo de seis espécies florestais, depositado em plantios puros e mistos, e do folhedo de plantio misto, de capoeira e de floresta natural, pelo modelo exponencial de $1^{\mathrm{a}}$ ordem

\begin{tabular}{|c|c|c|c|c|}
\hline \multirow{2}{*}{ Tipo de folhedo } & \multicolumn{2}{|l|}{ Puro } & \multicolumn{2}{|c|}{ Misto } \\
\hline & k (ano-1) & $\mathbf{R}^{2}$ & k (ano-1) & $\mathbf{R}^{2}$ \\
\hline Pau-roxo & $-0,856(0,086)^{(1)} A b^{(2)}$ & $0,970 * *$ & $-1,102(0,106) \mathrm{Ba}$ & $0,973 * *$ \\
\hline Putumuju & $-0,883(0,244) A b$ & $0,814^{*}$ & $-0,986(0,260) A a$ & $0,828^{*}$ \\
\hline Arapati & $-0,951(0,173) A b$ & $0,909 *$ & $-0,492(0,103) \mathrm{BC}$ & $0,884^{*}$ \\
\hline Arapaçu & $-0,709(0,067) \quad A b$ & $0,974 * *$ & $-0,556(0,049) A C$ & $0,977 * *$ \\
\hline Claraíba & $-1,197(0,379) \mathrm{Aa}$ & 0,768 & $-1,210(0,112) \mathrm{Aa}$ & $0,974 * *$ \\
\hline Óleo-comumbá & $-0,449(0,070) A C$ & $0,932 * *$ & $-0,784(0,086) \mathrm{Bb}$ & $0,965^{* *}$ \\
\hline Médio(3) & $-0,810(0,050)$ & $0,989 * * *$ & $-0,818(0,092)$ & $0,963 * *$ \\
\hline Misto & $-0,816(0,040) b$ & $0,993 * * *$ & & \\
\hline Capoeira & $-0,648(0,184) b$ & $0,805^{*}$ & & \\
\hline Floresta natural & $-0,855(0,125) a$ & $0,940 * *$ & & \\
\hline
\end{tabular}

(1) Os valores entre parênteses correspondem ao desvio-padrão. ${ }^{(2)}$ Os valores seguidos pela mesma letra, maiúscula na linha e minúscula na coluna, não diferem entre si pelo teste t. ${ }^{(3)}$ Valor de $k$ estimado dos valores médios das espécies em plantios puros e no misto. *, ** e***: Significativos a 5,1 e $0,1 \%$ pelo teste $\mathrm{F}$, respectivamente. 
Pau-roxo

- misto $\hat{y}=96,921 e^{-1,1025 x} R^{2}=0,97^{\star *}$

… . . puro $\hat{y}=93,189 e^{-0,8557 x} R^{2}=0,97^{\text {** }}$

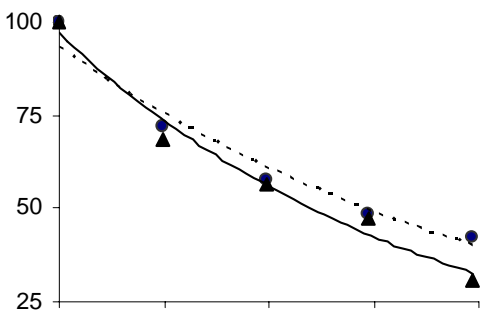

Arapati
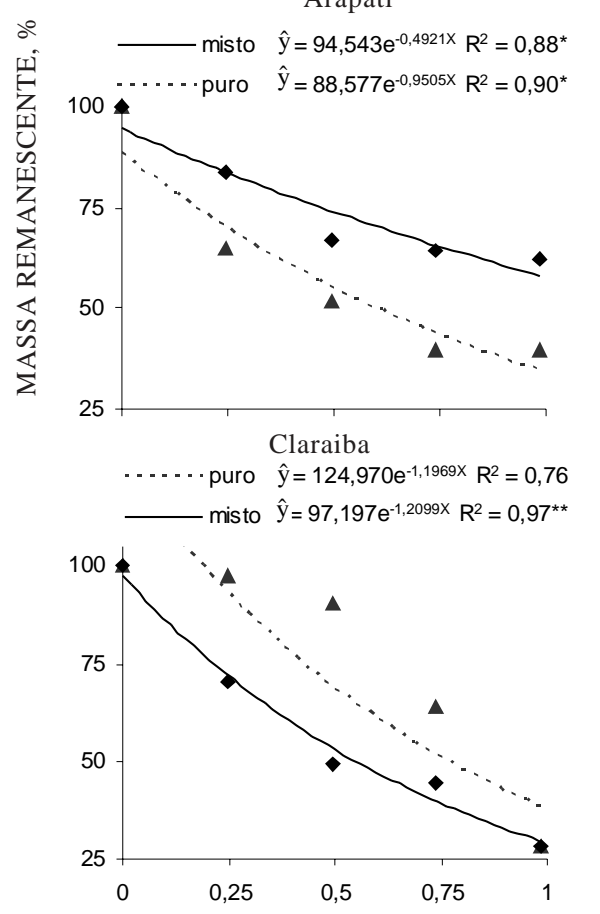

Putumuju

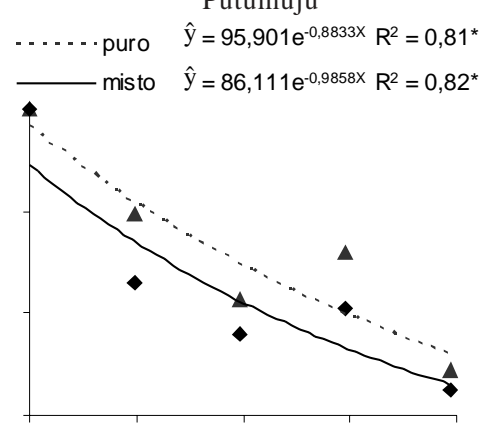

Arapaçu
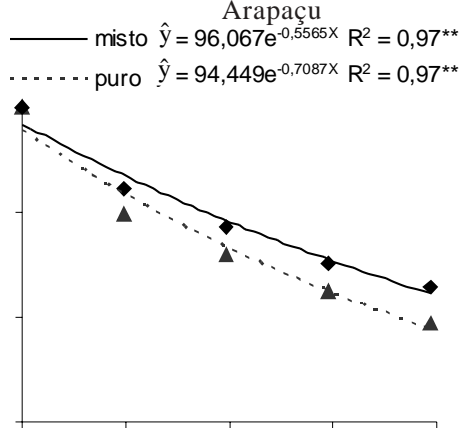

Óleo-comumbá

puro $\hat{y}=95,551 e^{-0,4493 x} R^{2}=0,93^{\star *}$

misto $\hat{y}=95,351 e^{-0,7838 x} R^{2}=0,96^{\star \star}$

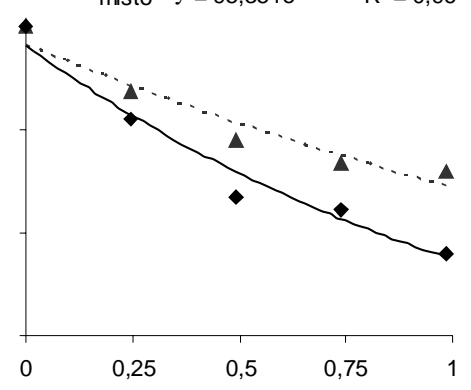

ANO

. . . . . misto $\quad \hat{y}=99,421 e^{-0,816 x} R^{2}=0,99^{\star \star *}$

- - - capoeira $\hat{y}=87,239 e^{-0,6479 x} R^{2}=0,80^{*}$

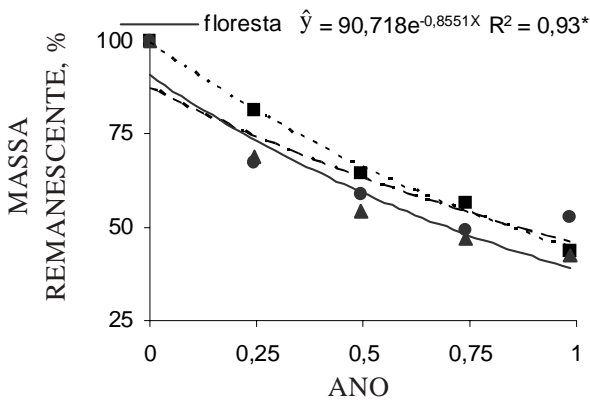

Figura 1. Percentual de massa remanescente do folhedo de seis espécies florestais em decomposição, depositado em planti os puros e mistos, e do fol hedo de plantio misto, de capoeira e de floresta natural. *, ** e**: significativos a 5,1 e $0,1 \%$ pelo teste $F$, respectivamente.

magnitude de interação desses substratos com os componentes da serapilheira daquele ecossistema, já que todos os tipos defol hedo estão aparentemente sob o mesmo microambiente.

Dentre os ecossistemas heterogêneos, a taxa de decomposição do folhedo do plantio misto diferiu significativamente apenas da floresta natural (Quadro 2 e Figura 1). Esperar-se-ia que os três ecossistemas tivessem taxas similares graças ao el evado número de espécies que os compõem, sob as mesmas condições edafoclimáticas. Numa simulação, os valores médios das taxas de 
decomposição do fol hedo das espécies em estudo, seja em plantios puros, seja no misto, foram similares aos da taxa do próprio plantio misto (Quadro 2). Esses resultados evidenciam que a taxa de decomposição do folhedo do plantio misto ficaria numa posição intermediária em relação às taxas das espécies contrastantes que compõem este ecossistema.

Resultados similares foram encontrados por Blair et al. (1990), ao aval iarem as taxas de decomposição de Cornus florida, Acer rubrum e Quercus primus em plantios puros e mistos, considerando as misturas, dupla ou tripla, dessas espécies. Nesse sentido, as taxas de decomposição dos fol hedos da capoeira e da floresta natural provavelmente expressariam também valores médios das taxas individuais das espécies que compõem esses ecossistemas (Quadro 2).

Para as espécies florestais e os três ecossistemas heterogêneos, os maiores valores dos teores de $\mathrm{N}$ e $\mathrm{P}$, ao final do período de decomposição, poderiam ser decorrentes da maior perda relativa de carbono (Quadro 3). A liberação de N e P do fol hedo de cada espécie variou de acordo com o microambiente (Figuras 2 e3). Em relação ao N, em termos médios, não houve diferença significativa entre os dois sistemas de plantio (microambiente) para o pau-roxo, arapati, arapaçu e óleo-comumbá, não ocorrendo, também para estas três últimas espécies, diferença significativa para oP. O importante, contudo, éavaliar o fluxo do N e P durante o período de decomposição.
Nesse sentido, a liberação desses nutrientes do folhedo do putumuju e claraíba distinguiu marcadamentea influência dos dois mi croambientes, sendo observado maior fluxo no plantio misto (Figuras 2 e3). Destaca-sea acumulaçãolíquida de $\mathrm{N}$ eP na claraíba, em plantio puro, durantea maior parte do período de decomposição. A penas no pauroxo, em plantio puro, ocorreu maior fluxo de $\mathrm{P}$ até os 270 dias de decomposição (Figura 3). No putumuju e no óleo-comumbá, de maneira geral, ocorreram as mai ores e menores, respectivamente, liberações do $\mathrm{N}$ eP, em ambos os sistemas de plantio (Figuras 2 e 3).

Dentre os ecossistemas heterogêneos, a maior liberação do N ocorreu no plantio misto, diferindo significativamente, em termos médios, da capoeira eda floresta natural (Figura 2). J á para oP, a capoeira foi o ecossistema que apresentou significativamente a menor li beração (F igura 3).

Houve aumentos dos teores de lignina, de maneira geral, ao final do período de decomposição (Quadro 3). A penas no putumuju ocorreu decréscimo em ambos os sistemas de plantio. Entretanto, houve redução dos teores de celulose, à exceção no putumuju, claraíba e óleo-comumbá em plantios puros (Quadro 3). Os teores de lignina geralmente aumentam nos estádios mais avançados de decomposição (Rowland \& Roberts, 1994; Berg, 2000), enquanto os de celulose diminuem (Heal et al., 1997).

Quadro 3. Atributos quími cos do fol hedo de seis espécies florestais, depositado em plantios puros e mistos, e do folhedo de plantio misto, de capoeira e de floresta natural

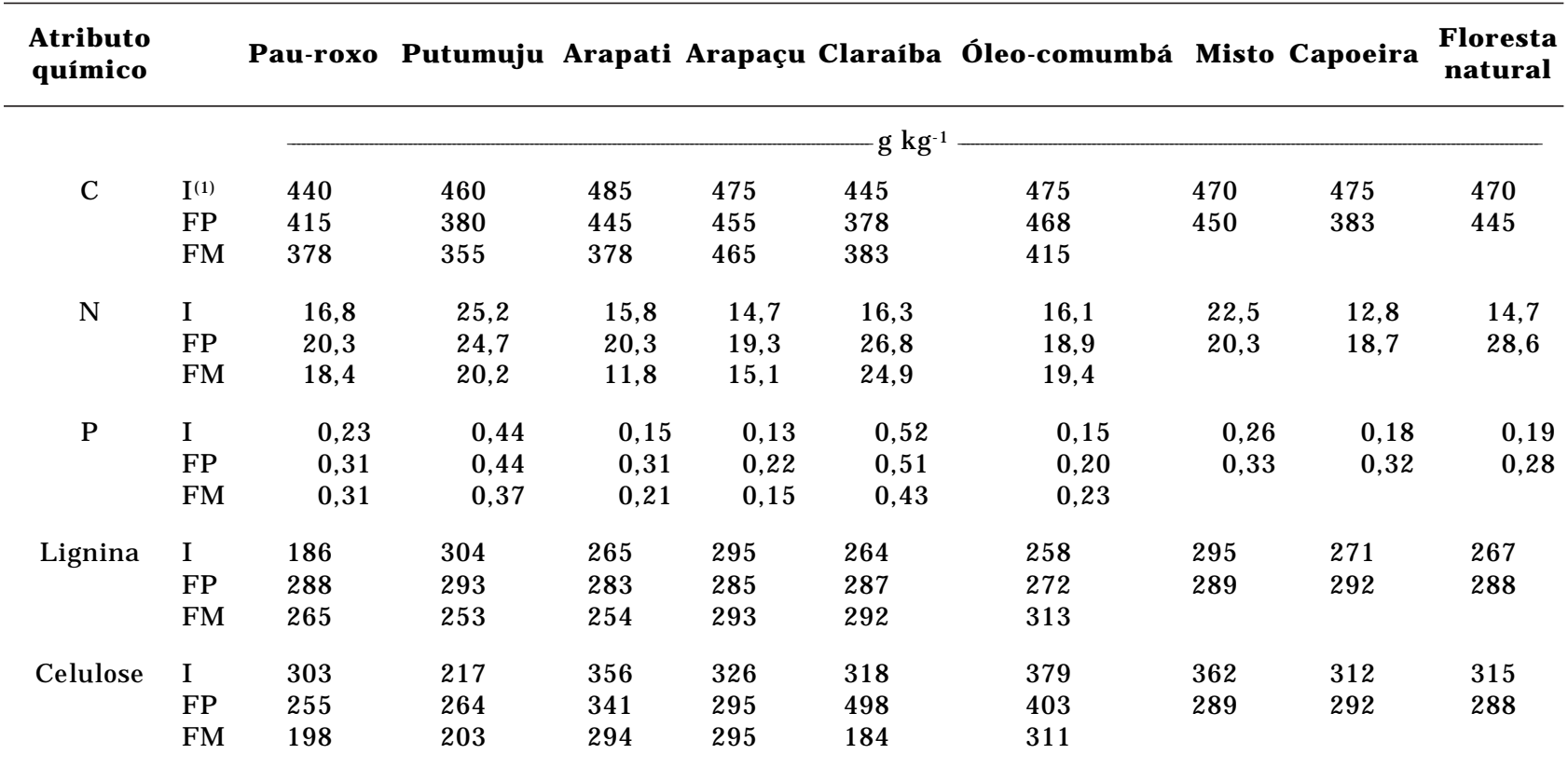

$\overline{(1)}$ I = Peso do material seco inicial; FP = Peso do material seco remanescente final no plantio puro; FM = Peso do material seco remanescente final no plantio misto. 


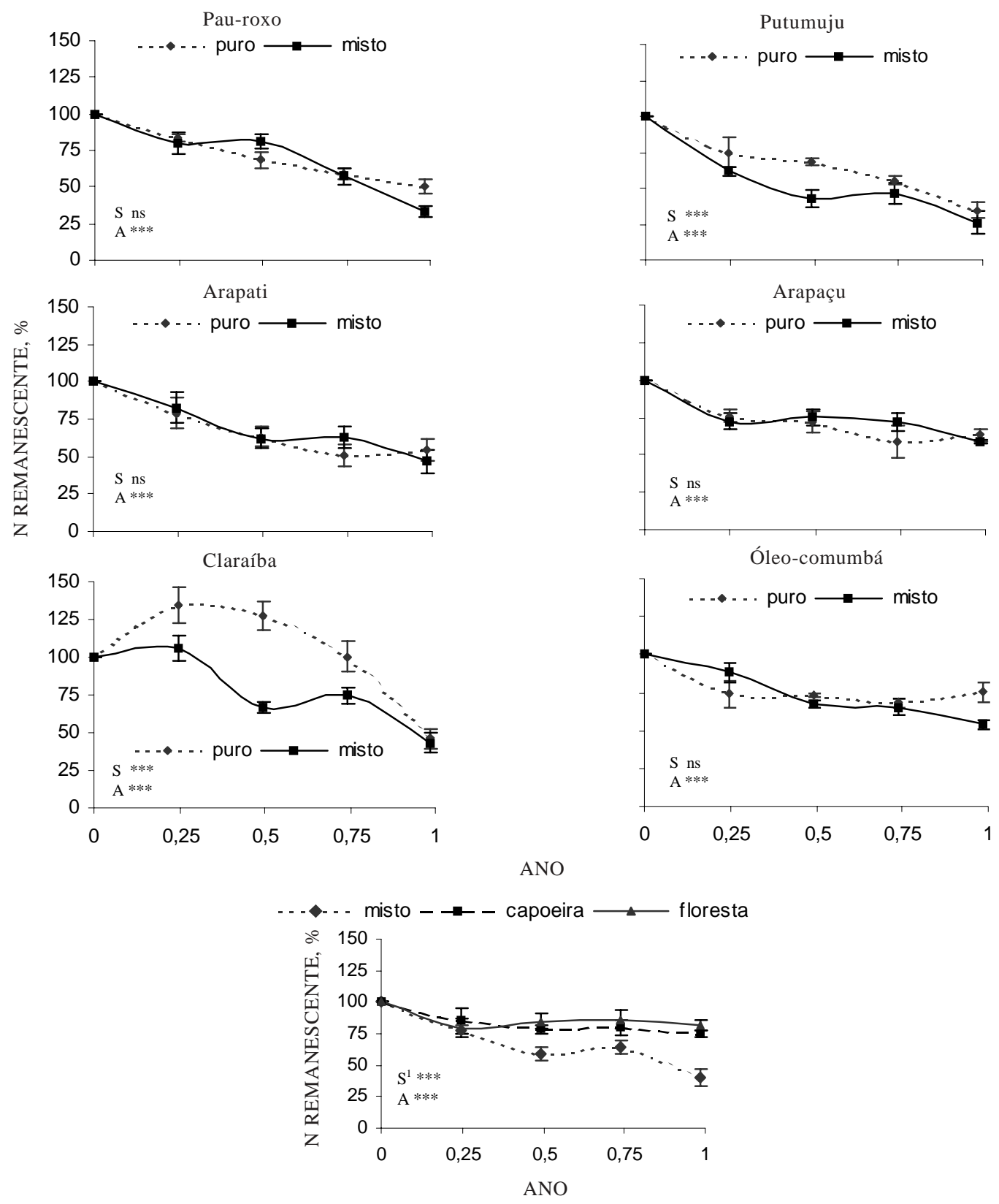

Figura 2. Percentual de massa remanescente de $\mathbf{N}$ do folhedo de seis espécies florestais, depositado em plantios puros e mistos, e do folhedo de plantio misto, de capoeira e de floresta natural. As barras verticais correspondem ao erro-padrão. S: sistemas de plantio puro X misto; A: ano. ** e***: significativos a 1 e $0,1 \%$ pelo teste $F$, respectivamente. $S^{1}$ : sistemas de plantio misto $X$ capoeira $X$ floresta.

Esses resultados são indícios de que as interações dos fatores abióticos e bióticos, que regulam a funcionalidade dos ecossistemas florestais, atuariam positiva ou negativamente na velocidade de decomposição do fol hedo das espécies florestais, mostrando, assim, que, mesmo em nível local, o processo de decomposição não seria influenciado apenas pela qualidade do substrato, mas também pela qualidade do microambiente.

A umidade do fol hedo seria um fator de qualidade importante no processo de decomposição. Na coleta dos sacos de decomposição, observou-se maior umidade nos folhedos das espécies florestais no plantio misto, mesmo nos períodos de baixa precipitação. Situação semel hante foi reportada por Gama-Rodrigues \& Barros (2002), quando depositaram fol hedo de eucalipto (E. grandis $x E$. urophylla) em fragmentos de floresta natural, verificando, neste ecossistema, maiores perdas de massa $(52,5 \%)$ do fol hedo daquela espécie do que no próprio povoamento de eucalipto $(42,6 \%)$. Sankaram (1993) encontrou correlações positivas 


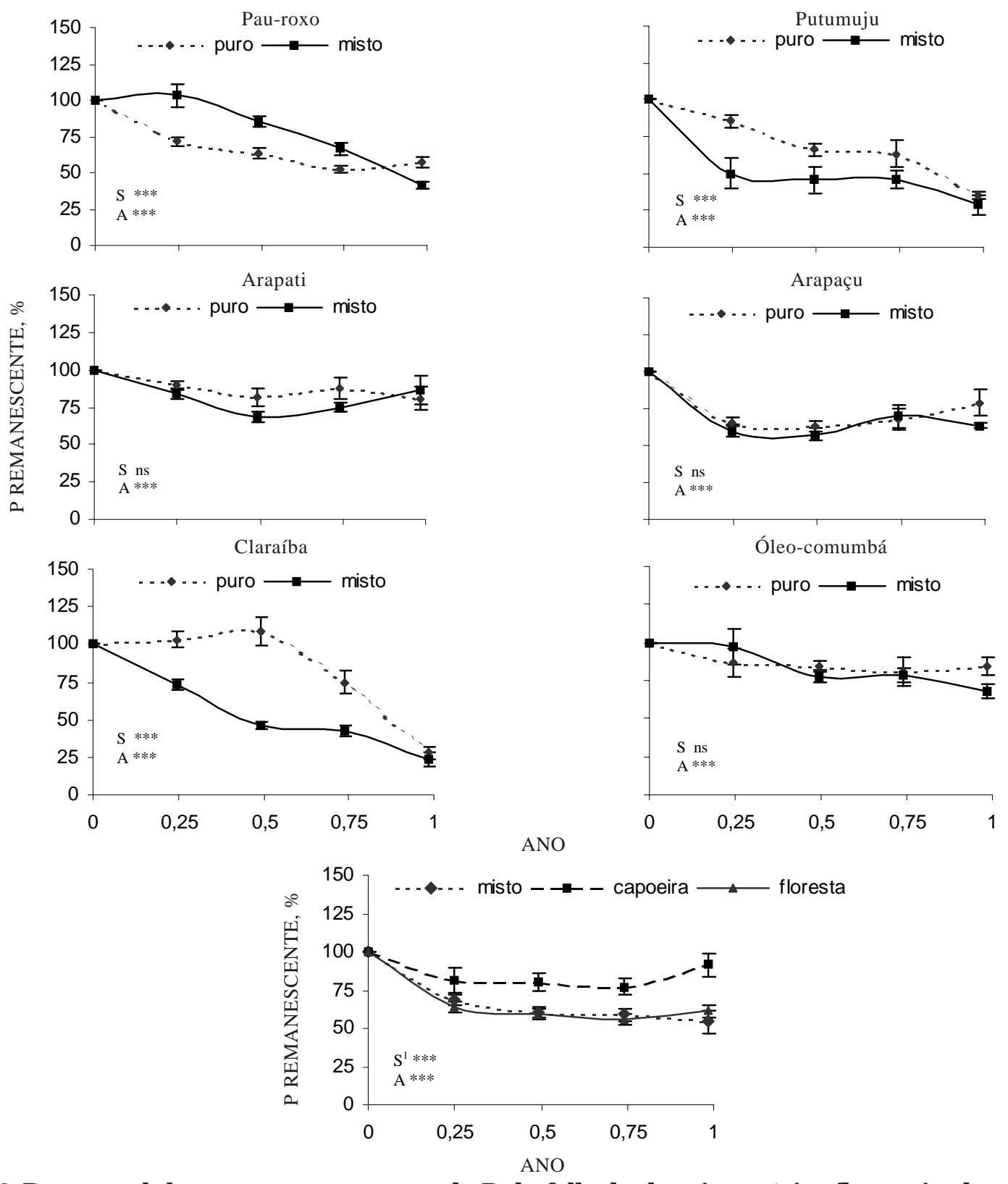

Figura 3. Percentual de massa remanescente de $P$ do folhedo de seis espécies florestais, depositado em plantios puros e mistos, e do folhedo de plantio misto, de capoeira e de floresta natural. As barras verticais correspondem ao erro-padrão. S: sistemas de plantio puro X misto; $\mathrm{A}$ : anos. ** $\mathrm{e}^{* * *}$ : significativos a 1 e $0,1 \%$ pelo teste $F$, respectivamente. $S^{1}$ : sistemas de plantio misto $X$ capoeira $X$ floresta.

entre a perda de peso e o teor de umidade dos fol hedos de eucalipto (E. tereticornis), teca (Tectona grandis) e al bizia (Parasenianthes fal cataria).

No plantio misto, por outro lado, podem ter ocorrido, também, interações positivas dos fol hedos do pau-roxo e óleo-comumbá com os componentes da serapilheira daquele ecossistema, acelerando a decomposição dos fol hedos dessas espécies florestais. Briones \& I neson (1996) observaram aumentos da decomposição do fol hedo de E. gl obulus, quando misturado (dois a dois) com fol hedos deQuercus petraea, Fraxanus excelsior e Betula pendula. Interação positiva também foi verificada para a mistura do fol hedo Populus tremuloides com Alnus crispa (Binkley, 1992). FolhedodeCordia alliodora sedecompõe mais rapidamente em plantio misto com outras espécies arbóreas do que em plantio puro (Babbar \& E wel, 1989). Entretanto, K lemmedson (1992) não encontrou efeito positivo sobre a decomposição de Pinus ponderosa misturado em diversas proporções com fol hedo de Quercus gambilii. Köchy \& Wilson (1997) reportaram que a deposição do fol hedo de Populus tremuloi des em pastagem natural nãoalterou a taxa de decomposição dessa espécie. 
No plantio misto, o aporte contínuo de folhedo expressaria uma propriedade coletiva dos indivíduos de várias espécies, de composi ções químicas distintas, que ocorrem no sítio, enquanto as taxas de decomposição da serapilheira revelariam uma propriedade emergente, em virtude não só da interação dos processos de decomposição de seus componentes, mas também do somatório das taxas individuais das espécies (Gama-Rodrigues, 1997). O plantio misto, portanto, reproduziria certa complexidade de interações, assemelhando-se às florestas naturais. Nestes ecossistemas, as propriedades col etivas e emergentes manifestar-se-iam simultaneamente (Salt, 1979; Odum, 1983; Yost et al., 1997).

Nesse sentido, a liberação e, ou, enriquecimento líquido de $\mathrm{N}$ eP dos fol hedos das espécies florestais alocados no plantio misto e dos três ecossistemas heterogêneos pode ser atribuída à movimentação interespecífica de nutrientes ocorrida em folhedos heterogêneos, governada pelo gradiente de nutrientes (Briones \& Ineson, 1996). Situação anál oga foi reportada por Wood (1974), que observou aumentos do teor de $\mathrm{N}$ durante a decomposição do fol hedo de E. del egatensis, mediantea transl ocação do el emento pelas hifas de fungos. Esse mecanismo também foi proposto em relação à acumulação de $\mathrm{P}$ durantea decomposi ção dos fol hedos de E. gl obul us, Cupressus Iusitanica, J uni perus procera eda floresta natural altomontana da Etiópia (Lisanework \& Michelsen, 1994). A fauna ea lixiviação do fol hedo, também, são importantes fatores de transferência interespecífica de nutrientes (Heal et al., 1997).

Os val ores das características referentes ao início do estudo (Quadro 3) apresentaram baixa capacidade preditiva da perda de peso do fol hedo, principalmente em plantios puros (Quadro 4). No plantio misto, apenas os teores de C eP, a relação C/P eas respectivas combinações dos teores de $\mathrm{P}$ com os de lignina e celulose, mostraram relação linear significativa com a massa remanescente final do fol hedo. Também, apenas no plantio misto a regressão linear múltipla, com os teores de C e P, aumentou a capacidade preditiva da massa remanescente final do fol hedo $\left[\% M R=-214,08-47,35(P)+0,577(C) ; R^{2}=0,927 *\right]$.
Esses resultados revelam que o $P$, e não o $N$, seria mais limitante para a decomposição do fol hedo dessas espécies, nas condições estudadas. Para vários tipos de coberturas florestais, em solos altamente intemperizados, o $\mathrm{P}$ se mostra como principal fator nutricional limitante da decomposição do folhedo (Attiwill \& Adams, 1993). Esses resultados indicam, aparentemente, a influência dos dois sistemas de plantio (microambiente) sobre os atributos de qualidade que regulam o processo de decomposição do folhedo. Assim, a capacidade preditiva desses atributos não seria independente do tipo de microambiente no qual o substrato se decompõe. I sso possibilita, então, quea decomposição do folhedo de espécies florestais com diferentes características químicas seja utilizada como indicador para distinguir qualidade de sítios florestais e, ou, sistemas de produção florestal.

Os resultados de decomposição e liberação de nutrientes, observados no presente trabalho, revelam o potencial de mani pulação da serapilheira dos ecossistemas florestais estudados. Em fol hedos heterogêneos, a transferência de nutrientes do material dealta qualidade para o de baixa qualidade afetaria a disponibilidade dos el ementos, pela maior conservação no sistema solo-serapilheira, e possivel mente el evaria a taxa de decomposição do substrato mais recalcitrante, alterando subseqüentemente a qualidade da matéria orgânica do solo (Heal et al., 1997; Handayanto et al., 1997). Nesse sentido, o plantio misto de espécies nativas com folhedos de qualidades bastante distintas seria um sistema ecológico que aumentaria a eficiência da ciclagem de matéria orgânica e de nutrientes, reproduzindo, em certo limite, as condições ecofisiológicas da floresta natural.

\section{CONCLUSÕES}

1. A taxa de decomposição do fol hedo do plantio misto situa-se numa posição intermediária em relação às taxas das espécies contrastantes que compõem este ecossistema. Isto proporciona maior

Quadro 4. Regressões lineares da massa remanescente final do folhedo de seis espécies florestais de acordo com seus atributos químicos, depositado em plantios puros e mistos

\begin{tabular}{|c|c|c|c|c|c|c|}
\hline \multirow{2}{*}{ Variável } & \multicolumn{3}{|c|}{ Puro } & \multicolumn{3}{|c|}{ Misto } \\
\hline & Intercepto & Declividade & $\mathbf{R}^{2}$ & Intercepto & Declividade & $\mathbf{R}^{2}$ \\
\hline \multicolumn{7}{|l|}{ (massa inicial) } \\
\hline $\begin{array}{l}\mathrm{C}, \mathrm{g} \mathrm{kg}^{-1} \\
\mathrm{P}, \mathrm{g} \mathrm{kg}^{-1} \\
\mathrm{C} / \mathrm{P} \\
\mathrm{Lig} / \mathrm{P} \\
(\mathrm{Lig}+\mathrm{Cel}) / \mathrm{P}\end{array}$ & $\begin{array}{r}-113,012 \\
57,939 \\
26,318 \\
26,333 \\
26,328\end{array}$ & $\begin{array}{c}0,337 \\
-54,774^{\circ} \\
0,007 \\
0,012 \\
0,006^{\circ}\end{array}$ & $\begin{array}{l}0,241 \\
0,550 \\
0,500 \\
0,454 \\
0,530\end{array}$ & $\begin{array}{r}-299,154 \\
61,114 \\
17,067 \\
16,801 \\
18,410\end{array}$ & $\begin{array}{r}0,737^{*} \\
-69,003^{\circ} \\
0,011^{*} \\
0,019^{*} \\
0,008^{*}\end{array}$ & $\begin{array}{l}0,818 \\
0,620 \\
0,809 \\
0,752 \\
0,769\end{array}$ \\
\hline
\end{tabular}

${ }^{\circ} \mathrm{e}^{*}$ : Significativos a 10 e $5 \%$ pelo teste $\mathrm{F}$, respectivamente. 
capacidade de reciclar a matéria orgânica e os nutrientes.

2. Os processos de decomposição e mineral ização são influenciados não apenas pela qualidade individual do substrato, mas também pela qualidade do microambiente (interação entre fatores físicoquímicos e a biota decompositora) de determinado sistema de plantio.

3. A extrapolação de resultados desses processos obtidos em cultivos monoespecíficos para sistemas florestais heterogêneos não é apropriada.

\section{LITERATURA CITADA}

ANDERSON, J.N. \& INGRAM, J .S.I. Tropical soil biology and fertility: A handbook of methods. Wallingford, CAB International, 1989. 171p.

ATTIWILL, P.M. \& ADAMS, M.A. Nutrient cycling in forests. New Phytol., 124:561-582, 1993.

BABBAR, L.I. \& EWEL, J.J. Decomposicion del follaje en diversos ecosistemas sucesionales tropicales. Biotropica, 21:21-29, 1989.

BATAGLIA, O.C.; FURLANI, A.M.C.; TEIXEIRA, J.P.F.; FURLANI, P.R. \& GALLO, J.R. Métodos de análise química de plantas. Campinas, Instituto Agronômico de Campinas, 1983. 48p. (Boletim Técnico, 78)

BERG, B. Litter decomposition and organic matter turnover in northern forest soil. For. Ecol. Manag., 133:13-22, 2000.

BRAGA, J .M. \& DEFELIPO, B.V. Determinação espectrofotométrica de fósforo em extrato de sol o e material vegetal. $R$. Ceres, 21:73-85, 1974.

BINKLEY, D. Mixtures of nitrogen $2^{-}$fixing and non-nitrogen $2^{-}$ fixing tree species. In: CANNELL, M.G.R.; MALCOLM, D.C. \& ROBERTSON, P.A., eds. The ecology of mixedspecies stands of trees. Oxford, The British Ecological Society, 1992. p.99-123.

BLAIR, J .M.; PARMELEE, R.W. \& BEARE, M.H. Decay rates, nitrogen fluxes, and decomposer communities of single and mixed-species foliar litter. E cology, 71:1076-1985, 1990.

BRIONES, M.J.I. \& INESON, P. Decomposition of eucalyptus leaves in litter mixtures. Soil Biol. Biochem., 28:1381-1388, 1996.

CORREIA, M.E.F . \& ANDRADE, A.G. Formação de serapilheira e ciclagem de nutrientes. In: SANTOS, G.A. \& CAMARGO, F.A.O., eds. Fundamentos da matéria orgânica do solo: ecossistemas tropicais e subtropicais. PortoAlegre, Gênesis, 1999. p.197-225.

GAMA-RODRIGUES, A.C. Ciclagem de nutrientes por espécies florestais em povoamentos puros e mistos em solos de tabuleiro da Bahia, Brasil. Viçosa, Universidade Federal de Viçosa, 1997. 107p. (Tese de Doutorado)

GAMA-RODRIGUES, A.C.; BARROS, N.F \& MENDONÇA, E.S. Alterações edáficas sob plantios puros e misto de espécies florestais nativas do sudeste da Bahia, Brasil. R. Bras. Ci. Solo, 23:581-592, 1999.
GAMA-RODRIGUES, A.C. \& BARROS, N.F. Ciclagem de nutrientes em floresta natural e em plantios de eucalipto e de dandá no sudeste da Bahia, Brasil. R. Árvore, 26:193207, 2002.

HANDAYANTO, E.; GILLER, K.E. \& CADISCH, G. Regulating $\mathrm{N}$ release from legume tree prunings by mixing residues of different quality. Soil Biol. Biochem., 29:1417-1426, 1997.

HEAL, W.;ANDERSON, J .M. \& SWIFT, M.J . Plant litter quality and decomposition: An historical overview. In: CADISCH, G. \& GILLER, K.E., eds. Driven by nature: Plant litter quality and decomposition. Wallingford, CAB International, 1997. p.3-30

KLEMMEDSON, J .O. Decomposition and nutrient release from mixtures of Gambel oak and ponderosa pine leaf litter. For. Ecol. Manag., 47:349-361, 1992.

KÖCHY, M. \& WILSON, S.D. Litter decomposition and nitrogen dynamics in aspen forest and mixed-grass prairie. Ecology, 78:732-739, 1997.

LAVELLE, P.; BLANCHART, E.; MARTIN, A. \& MARTIN, S. A hierarchical model for decomposition in terrestrial ecosystems: applications to soils of humid tropics. Biotropica, 25:130-150, 1993.

LEÃO, A.C. \& MELO, A.A.O. Características morfológicas, físicoquímicas e mineralógicas dos solos da Estação Ecológica do Pau-Brasil, PortoSeguro, Bahia. Agrotrópica, 2:105-112, 1990.

LISANEWORK, N. \& MICHELSEN, A. Litterfall and nutrient release by decomposition in three plantations compared with a natural forest in the Ethiopian highland. For. Ecol. Manag., 65:149-164, 1994.

MCCLAUGHERTY, C.A.; PASTOR, J .; ABER, J.D. \& MELILLO, J .M. Forest litter decomposition in relation to soil nitrogen dynamics and litter quality. E cology, 66:465-472, 1985.

MELILLO, J .M.; ABER, J .D. \& MURATORE, J .F. Nitrogen and lignin control of hardwood leaf litter decomposition dynamics. Ecology, 63:621-626, 1982.

MORI, S.A. \& SILVA; L.A.M. O herbário do Centro dePesquisas do Cacau em Itabuna, Brasil. Ilhéus, Bahia, Brasil. Comissão Executiva do Plano da Lavoura Cacaueira/Centro de Pesquisas do Cacau, 1980. 8p.(Boletim Técnico, 78)

ODUM, E.P. The strategy of ecosystems development. Science, 164:262-270, 1969.

ODU M, E.P. E cologia. Rio deJ aneiro, Guanabara Koogan, 1983. $434 p$.

OLSON, J .S. Energy storage and the balance of producers and decomposers in ecological systems. Ecology, 44:322-331, 1963.

ROWLAND, A.P. \& ROBERT, J.D. Lignin and cellulose fractionation in decomposition studies using acid-detergent fibre methods. Commun. Soil Sc. Plant Anal., 25:269-277, 1994.

SALT, G.W. A comment on use of the use of the term "emergent properties". Am. Nat., 113:145-148, 1979. 
SANKARAN, K.V. Decomposition of leaf litter of albizia (Paraserianthes falcataria), eucalypt (Eucalyptus tereticornis) and teak (Tectona grandis) in Kerala, India. For. Ecol. Manag., 56:225-242, 1993.

SCHLESINGER, W.H. \& HASEY, M.M. Decomposition of chaparal shrub foliage: losses of organic and inorganic constituents from deciduous and evergreen leaves. Ecology, 62:762-774, 1981.

TAYLOR, B.R.; PARKINSON, D. \& PARSONS, W.F.J . Nitrogen and lignin content as predictors of litter decay rates: a microcosm test. Ecology, 70:97-104, 1989.

van SOEST, P. \& WINE, R.H. Development of a comphrehensive system of feed analysis and its applications to forages. J . Assoc. Off. Agr. Chem., 51:780-785, 1968.

van WESEMAEL, B. Litter decomposition and nutrient distribution in humus profiles in some mediterranean forests in southern Tuscany. For. E col. Manag., 57:99-114, 1993.

VELOSO, H.P.; RANGEL FILHO, A.L.R. \& LIMA, J.C.A. Classificação da vegetação brasileira adaptada a um sistema universal. Rio de J aneiro, Instituto Brasileiro de Geografia e Estatística, 1991. 124p.
VINHA, S.G. \& LOBÃO, D.E.V.P. Estação ecológica do PauBrasil, Porto Seguro, Bahia. Ilhéus, Comissão Executiva do Plano da Lavoura Cacaueira/Centro de Pesquisas do Cacau, 1989. 40p.

WIEDER, R.K. \& LANG, G.E. A critique of the analytical methods used in examining decomposition data obtained from litter bags. Ecology, 63:1636-1642, 1982.

WOOD, T.G. Field investigations on the decomposition of leaves of Eucalyptus delegatensis in relation to environmental factors. Pedobiologia, 14:343-371, 1974.

YOST, R.; CALDWELL, R.; CONSTANTINIDES, M.; HERBERT, D. \& FOWNES, J. The sustainability of agriculture and forestry production systems on soils with low $\mathrm{pH}$. In: MONIZ, A.C.; FURLANI, A.M.C.; SCHAEFFERT, R.E.; FAGERIA, N.K.; ROSOLEM, C.A. \& CANTARELLA, H., eds. Plant-Soil interactions at low $\mathrm{pH}$ : sustainable agriculture and forestry production. Campinas/Viçosa, Brazilian Soil Sciency Society, 1997. p.29-38.

ZAR, J .H. Biostatistical analysis. 3.ed. New J ersey, PrenticeHall, Englewood Cliffs, 1996. 918p.

ZI MMER, M. Is decomposition of woodland leaf litter influenced by its species richness? Soil Biol. Biochem., 34:277-284, 2002. 
A.C. GAMA-RODRIGUES et al.

R. Bras. Ci. Solo, 27:1021-1031, 2003 